\title{
Raman spectroscopy and TGA studies for the synthesis of multi- walled carbon nanotube-functionalized thiol (MWCNT-SH) nanocomposites: study of effect of concentration
}

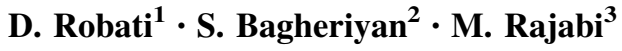

Received: 30 January 2015/Accepted: 7 May 2015/Published online: 30 May 2015

(c) The Author(s) 2015. This article is published with open access at Springerlink.com

\begin{abstract}
We have investigated some methods such as Raman spectroscopy and thermogravimetric analysis (TGA) studies for the nanocomposites multi-walled carbon nanotube-functionalized thiol (MWCNT-SH) by adding to cysteamine with known concentration in nanocomposite. For this study, we synthesized nanocomposite MWCNTSH by the reaction between multi-walled carbon nanotubes-functionalized carboxyl and the cysteamine. For study of the effect of concentration, cysteamine with different concentrations was used (1, 3 and $5 \%$ weight) and it was called MWCNT-SH1, MWCNT-SH3 and MWCNTSH5, respectively. Then, the nanocomposite provided was characterized by Fourier transform infrared spectroscopy (FT-IR) and scanning electron microscope (SEM). The surface structure of nanocomposites can be analyzed and confirmed using Raman and FT-IR spectroscopy. Also, to investigate the thermal stability TGA was used. The study of TGA showed that nanocomposite MWCNT-SH had low thermal stability at temperatures more than $100{ }^{\circ} \mathrm{C}$.
\end{abstract}

Keywords Raman spectroscopy - MWCNT ·

Functionalized thiol $\cdot$ Cysteamine $\cdot$ TGA

D. Robati

d_robati@yahoo.com; robati@iiau.ac.ir

1 Department of Chemistry, Islamshahr Branch, Islamic Azad University, Islamshahr, Iran

2 Department of Science, Shahryar Branch, Islamic Azad University, Shahryar, Iran

3 Department of Chemistry, Arak Branch, Islamic Azad University, Arak, Iran

\section{Introduction}

Today, carbon nanotube (CNT) has attracted high attention in interdisciplinary areas, because of its unique empty tube structure and many outstanding electronic, optical and mechanical properties [1]. In applications of carbon nanotubes, it can be used as adsorbent alone or as nanocomposites with other substances. In comparison with classical adsorbents such as clay and activated carbon, CNT is more attractive because of its physicochemically favorable stability, structural and high selectivity diversity. Extensive experiments have been conducted on the adsorption of organic or inorganic contaminants on CNTs such as dioxin [2], $\mathrm{Cd}^{2+}[3], \mathrm{Zn}^{2+}$ [4], $\mathrm{Cr}^{6+}$ [5], $\mathrm{Cu}^{2+}$ [6], fluoride [7] and $\mathrm{Pb}^{2+}[8]$ from aqueous solution.

Cysteamine $\left(\mathrm{HSCH}_{2} \mathrm{CH}_{2} \mathrm{NH}_{2}\right)$ is a chemical compound. It is a degradation product of the amino acid cysteine and the simplest stable aminothiol [9]. Some applications of cysteamine are employed, as it readily oxidizes to the corresponding disulfide, to the hydrochloride salt, in the presence of air in the treatment of disorders of cysteine excretion [9].

One of the latest products and useful aspects of nanotechnology is the nanocomposite. The nanocomposite is a solid with multiphase material where one of the phases has one, two or three dimensions of lower than $100 \mathrm{~nm}$ [10], or structures having nanoscale repeat distances between the different phases that make up the material [10]. Nanocomposite multi-walled carbon nanotube-functionalized thiol (MWCNT-SH) can be used in the adsorption process and other applications in the industry.

In this work, we have synthesized nanocomposite MWCNT-SH. To produce this nanocomposite, we used multi-walled carbon nanotube-functionalized carboxyl and cysteamine with known concentrations. For the study of the 
effect of concentration, cysteamine with different concentrations (1, 3 and $5 \%$ weight) called MWCNT-SH1, MWCNT-SH3 and MWCNT-SH5, respectively, was used. The provided nanocomposite was characterized by Raman spectra, its FTIR structure was confirmed and the morphology of the surface was also confirmed by scanning electron microscope (SEM). To investigate the thermal stability, thermogravimetric analysis (TGA) was used for the synthesis of nanocomposites in this study.

\section{Materials and methods}

\section{Materials}

Multi-walled carbon nanotube-functionalized carboxyl (MWCNT-COOH)

Multi-walled carbon nanotubes functionalized carboxylic was provided by Nano Amor Nanostructured \& Amorphous Materials, Inc. (USA) with a purity of over $95 \%$. The average diameter was 1-2 nm; length was 5-30 nm and the SSA was $\sim 400 \mathrm{~m}^{2} / \mathrm{g}$. It was prepared by catalytic chemical vapor deposition (CVD).

\section{Cysteamine}

Cysteamine was provided by Sigma-Aldrich Co. (Germany) with a purity of over $98 \%$. For synthesis, the following specifications were used in this study: Cysteamine hydrochloride, CAS number, 156-57-0; linear formula, $\left(\mathrm{HSCH}_{2} \mathrm{CH}_{2} \mathrm{NH}_{2}\right) \cdot \mathrm{HCl}$; molecular weight, $113.61 \mathrm{~g} / \mathrm{mol}$.

\section{Preparation of nanocomposite multi-walled carbon nanotube-functionalized thiol (MWCNT-SH)}

Nanocomposite MWCNT-SH was prepared according to the following method: $80 \mathrm{mg}$ of dry powder of multiwalled carbon nanotube-functionalized carboxyl with $80 \mathrm{mg}$ of cysteamine hydrochloride was dissolved in ethanol. Then, $50 \mathrm{mg}$ of 1-ethyl (3,3 di-amino-propyl acetate) carbon-amide $99 \%$ (EDC) that was used for better reaction between graphene oxide and glycine (with purity $\geq 99 \%$ provided by Aldrich Company) was used. For the study of effect of concentration, cysteamine with different concentrations was used (1, 3 and $5 \%$ weight) and was called MWCNT-SH1, MWCNT-SH3 and MWCNT-SH5, respectively. Then, we added to the above mixture $30 \mathrm{mg}$ of (NHS) $99.9 \%$ (Aldrich Company). The above-mentioned compounds were exposed for a day at $55{ }^{\circ} \mathrm{C}$ and to prevent sticking, the particles were stored in an ultrasonic bath. The above mixture was placed in a shaker to get an even sample. After completion of the reaction, nanocomposite MWCNT-SH was separated by micro filter and washed with deionized water and ethanol for several times. So after several rinses, the $\mathrm{pH}$ of the output water was neutralized. Then the resulting mixture was placed in an oven at $80{ }^{\circ} \mathrm{C}$ for $48 \mathrm{~h}$ until its moisture was removed completely.

\section{Results and discussion}

\section{Characterization of nanocomposite multi-walled carbon nanotube-functionalized thiol (MWCNT-SH)}

Scanning electron microscope (SEM)

The SEM of multi-walled carbon nanotube-functionalized carboxyl is shown in Fig. 1. Based on Fig. 1, the active particles' dispersal is shown on the surface of multi-walled carbon nanotube-functionalized carboxyl.

\section{FTIR spectra}

The FTIR technique was used to examine the surface of the nanocomposite. FTIR spectroscopy for the nanocomposite MWCNT-SH is shown in Fig. 2 and confirms the new chemical bands in the nanocomposite. For study of the effect of concentration, cysteamine with different concentrations was used (1, 3 and $5 \%$ weight) and it was called MWCNT-SH1, MWCNT-SH3 and MWCNT-SH5, respectively. As shown in Fig. 2, multi-walled carbon nanotube-functionalized thio (MWCNT-SH) nanocomposite in

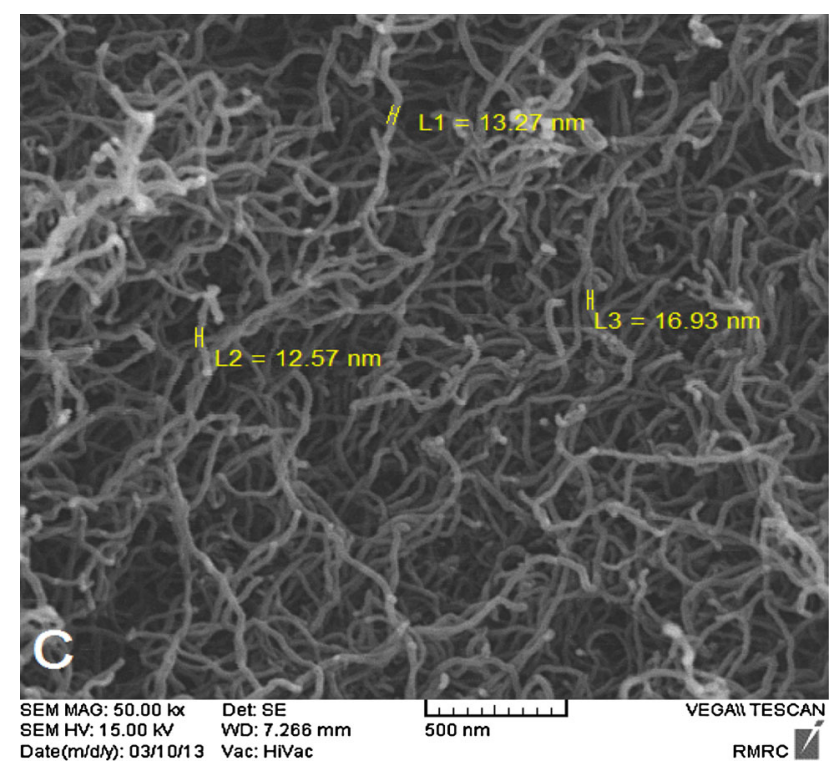

Fig. 1 SEM of multi-walled carbon nanotube-functionalized carboxyl 

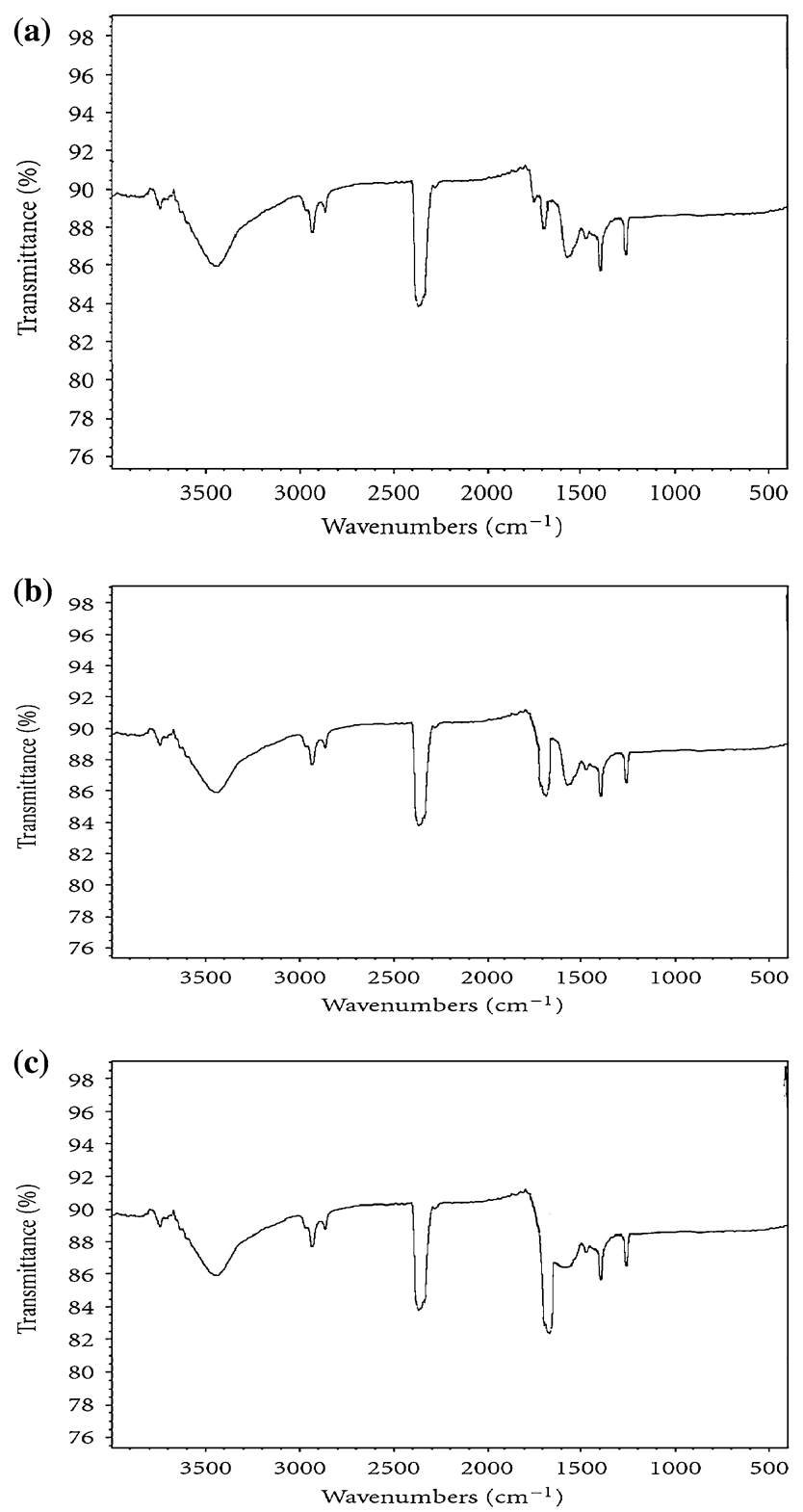

Fig. 2 FT-IR for nanocomposite multi-walled carbon nanotubefunctionalized thiol (MWCNT-SH). Cysteamine with different concentrations (1, 3 and $5 \%$ weight) called MWCNT-SH1, MWCNTSH3 and MWCNT-SH5, respectively

the $1634 \mathrm{~cm}^{-1}$ area was related to the amide group (CO$\mathrm{NH})$ which is a stretching bond. The peak at $2550 \mathrm{~cm}^{-1}$ was related to thiol functional groups( $\mathrm{SH})$ and the peak at 1350 was related to the bond that occurred by bending cysteamine [8].

\section{Raman spectroscopy study}

Raman spectroscopy of multi-walled carbon nanotubefunctionalized carboxyl MWCNT-COOH and nanocomposite MWCNT-SH are shown in Figs. 3 and 4. For more

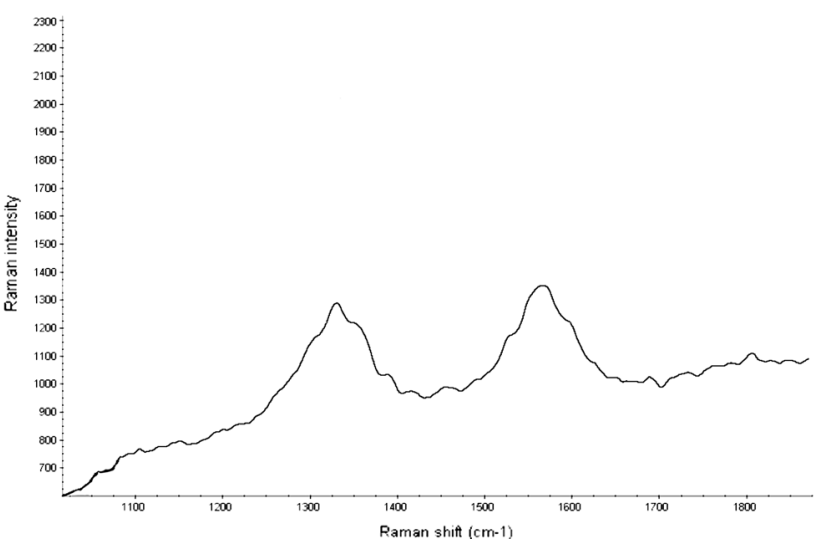

Fig. 3 Raman spectra of multi-walled carbon nanotube-functionalized carboxyl

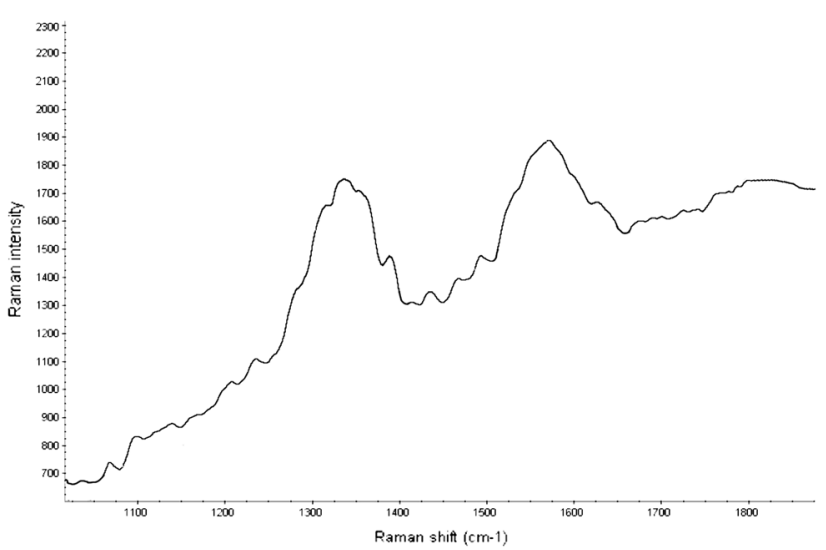

Fig. 4 Raman spectra of nanocomposite multi-walled carbon nanotube-functionalized thiol (MWCNT-SH)

control, functionalized with this chemical reaction has been confirmed by Raman spectroscopy. This method is a powerful way to identify functionalized carbon nanotubes. Bands G (1560-1580) and D (1330-1350) $\mathrm{cm}^{-1}$, respectively, can be seen clearly in the Raman spectra corresponding to MWCNT-COOH and MWCNT-SH in Figs. 3 and 4 which are attributed to tangential and induced irregular peaks,

Thermal stability of multi-walled carbon nanotubefunctionalized carboxyl MWCNT-COOH and nanocomposite multi-walled carbon nanotubefunctionalized thiol (MWCNT-SH)

The thermal behavior of multi-walled carbon nanotubefunctionalized carboxyl MWCNT-COOH and nanocomposite MWCNT-SH was studied by TGA at $600 \mathrm{c}$ by the SDTQ device, as shown in Figs. 5 and 6.

The temperature range was selected from 50 to $550{ }^{\circ} \mathrm{C}$ and the thermal velocity was $10{ }^{\circ} \mathrm{C} / \mathrm{min}$. 


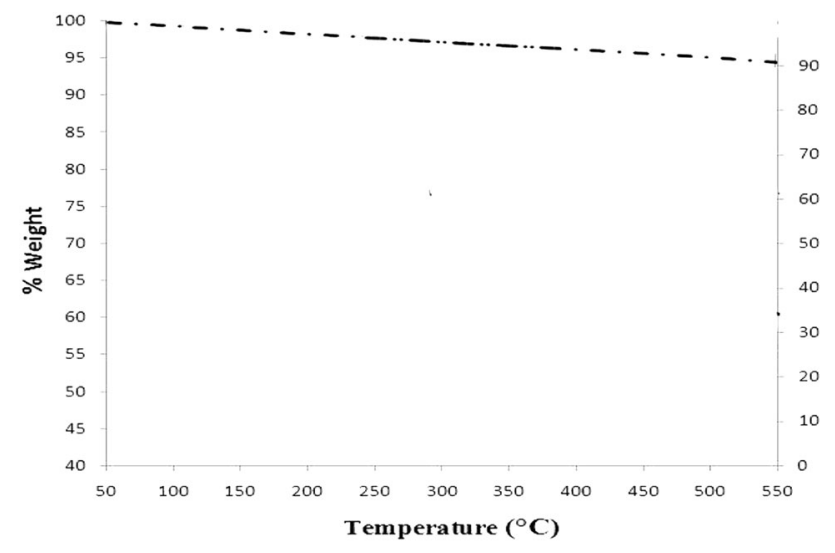

Fig. 5 Thermogravimetric analysis (TGA) for multi-walled carbon nanotube-functionalized carboxyl (MWCNT-COOH)

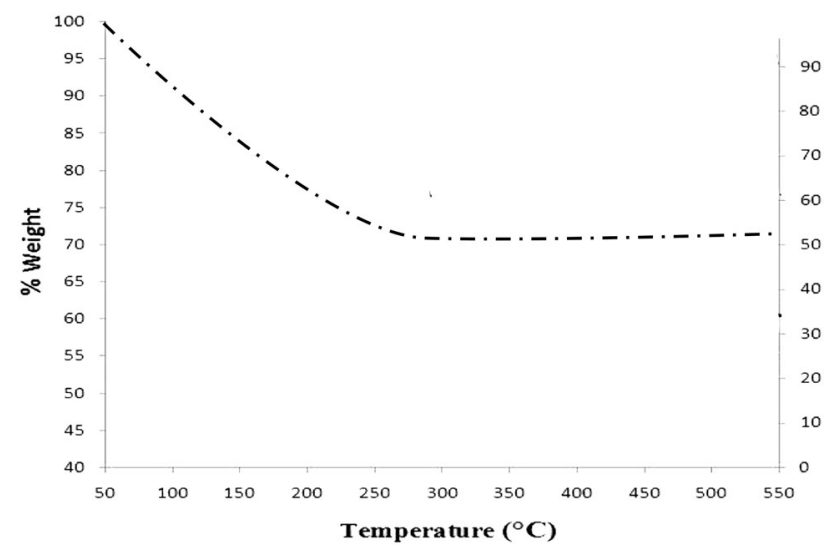

Fig. 6 Thermogravimetric analysis (TGA) for nanocomposite multiwalled carbon nanotube-functionalized thiol (MWCNT-SH)

As seen in Figs. 5 and 6, the multi-walled carbon nanotube-functionalized carboxyl (MWCNT-COOH) indicates relatively good and high thermal stability, but in Fig. 6 for nanocomposite MWCNT-SH low thermal stability at temperatures more than $100{ }^{\circ} \mathrm{C}$ was observed and may be related to amide groups composed on the surface of multiwalled carbon nanotube-functionalized carboxyl.

\section{Conclusions}

We synthesized nanocomposite MWCNT-SH by a reaction between dry powders of multi-walled carbon nanotubefunctionalized carboxyl and cysteamine as a new nanocomposite. Then we used infrared (IR) spectroscopy and SEM to characterize the surface of the nanocomposite. To analyze the structure of the surface, Raman spectra of the nanocomposite were investigated. Raman spectroscopic study of the synthesized nanocomposite showed that bands $\mathrm{G}(1560-1580)$ and $\mathrm{D}(1330-1350) \mathrm{cm}^{-1}$, respectively, can be seen clearly in the Raman spectra corresponding to MWCNT-COOH and MWCNT-SH on synthesis. Also, to investigate the thermal stability TGA was used.

Acknowledgments The author would like to thank the Islamic Azad, Islamshahr Branch, for their financial support.

Open Access This article is distributed under the terms of the Creative Commons Attribution 4.0 International License (http:// creativecommons.org/licenses/by/4.0/), which permits unrestricted use, distribution, and reproduction in any medium, provided you give appropriate credit to the original author(s) and the source, provide a link to the Creative Commons license, and indicate if changes were made.

\section{References}

1. Robat, D., Fakhri, A.: Modeling of the adsorption kinetics of Basic Red 46 on single-walled carbon nanotube and carboxylate group functionalized single-walled carbon nanotube. Phys. Theor. Chem. IAU Iran 9(2), 125-133 (2012)

2. Zhou, L., Zhang, X., Yang, H., Peng, B.: Adsorption and ability to carry catalysts of carbon nanotubes for destructing dioxins. Mater. Sci. 2, 226-231 (2009)

3. Li, Y., Wang, H., Luan, S., Ding, Z., Xu, J., Wu, C.D.: Adsorption of cadmium (II) from aqueous solution by surface oxidized carbon nanotubes. Carbon 41, 1057-1062 (2003)

4. Lu, C., Chiu, H.: Adsorption of zinc (II) from water with purified carbon nanotubes. Chem. Eng. Sci. 61, 1138-1145 (2006)

5. Lin, S., Lai, H., Leu, H.G.: Removal of heavy metals from aqueous solution by chelating resin in a multistage adsorption process. J. Hazard Mater. B76, 139-153 (2000)

6. Leng, Y., Guo, W., Su, S., Yi, C., Xing, L.: Removal of antimony(III) from aqueous solution by graphene as an adsorbent. Chem. Eng. J. 211-212, 406-411 (2012)

7. Li, Y., Wang, H.S., Wei, J., Zhang, X., Wei, J., Xu, C., Luan, Z., $\mathrm{Wu}, \mathrm{D}$.: Adsorption of fluoride from water by aligned carbon nanotubes. Mater. Res. Bull. 38, 469-476 (2003)

8. Li, Y.H., Wang, S., Wei, J., Zhang, X., Xu, C., Luan, Z., Wu, D., Wei, B.: Lead adsorption on carbon nanotubes. Chem. Phys. Lett. 357, 263-266 (2002)

9. Lukashin, B.P., Grebeniuk, A.N.: Comparative study of the radiation-protective effectiveness of low doses of cysteamine, heparin, and naphtizine in experiments on mice. Radiats. Biol. Radioecol. 41(3), 310-312 (2001)

10. Kamigaito, O.: What can be improved by nanometer composites. J. Jpn. Soc. Powder Powder Metall. 38(3), 315-319 (1991) 